\title{
Description of Interface Between Semiconductor Electrostatic Qubit and Josephson Junction in Tight Binding Model
}

\author{
K. POMORSKI ${ }^{a, b, c, *}$ AND R.B. StASZEWSKI ${ }^{d}$ \\ ${ }^{a}$ University College Dublin, School of Computer Science, Dublin, Ireland \\ ${ }^{b}$ Cracow University of Science and Technology, \\ Faculty of Computer Science and Telecommunications, Institute of Computer Science, \\ Warszawska 24, 31-155 Cracow, Poland \\ ${ }^{c}$ Quantum Hardware Systems (www.quantumhardwaresystems.com) \\ ${ }^{d}$ University College Dublin, School of Electrical and Electronic Engineering, Dublin, Ireland \\ Doi: 10.12693/APhysPolA.138.728 *e-mail: kdvpomorski@gmail.com
}

\begin{abstract}
The interface between a superconducting Josephson junction and a semiconductor position-based qubit implemented in coupled semiconductor $q$-dots is described as a possible base for an electrostatic interface between superconducting and semiconductor quantum computers. A modification of an Andreev bound state in a Josephson junction by the presence of a semiconductor qubit in its proximity and electrostatic interaction with a superconducting qubit is spotted by the minimalist tight-binding model. The obtained results allow for the creation of an interface between a semiconductor quantum computer and a superconducting quantum computer. The existence of topological states of matter is indicated. They open a perspective for the construction of QISKIT-like software that will describe both types of quantum computers as well as their interface.
\end{abstract}

topics: semiconductor qubit, Josephson junction interface, topological phase transitions, hybrid semiconductor-superconducting quantum computer

\section{Introduction}

Nature largely depends on mass and electric charge conservation principles. Both of these physical quantities are quasi-continuous on the macroscale and on the nanoscale they become the integer multiplicity of elementary values. The charge flows in such a way that the energy of the electric/magnetic field tends to be minimized. One of the consequences is the repulsion of two charges having the same sign and the attraction of two charges of the opposite signs. This is commonly known as Coulomb's law. The electric currents flow also tends to minimize the energy of magnetic field, therefore the most equilibrium state of an isolated capacitor is a discharged device. Due to electron and hole mobility, charge can be used for information or energy transfer across metallic or semiconductor nanowires. One can use the electric and magnetic fields as parameters controlling the evolution of the given physical system with time. Hence, the desired final state can be achieved upon setting the system with an initial configuration that is formally expressed by circuit theory, both in the classical and in quantum regime.

The simple rules of dynamics of charged billiard balls confined in boxes can lead to a simple scheme for the implementation of logical operations as a logical inverter or controllable inverter (CNOT gate), as depicted in Fig. 1 and in [1-6]. However, the electric charge is confirmed to be quantized by experiments (except for the fractional quantum Hall effect where a fractionation of electric charge is observed) and expressed by electron, proton or hole charge in condensed matter systems. The quantization and control of a single electron flow by distinct integer values can be achieved in nanotechnological experiments, e.g., in the chain of coupled quantum dots that can have particularly small diameters in semiconductors and in most recent CMOS technology - so even the size of $3 \mathrm{~nm}$ can be achieved for very highly integrated circuits. In such types of structures, the use of magnetic fields is less practical since waveguides and solenoids are very hardly scalable. Therefore, it is favourable to use only electric fields as a controlling factor. Here, the situation promotes Wannier qubits that are also known as position-based qubits. Wannier qubits use maximum localized wavefunctions present in two coupled quantum dots in order to encode quantum information in a qubit which makes such a qubit different from an eigenenergy-based qubit using two eigenenergies to span the qubit state. However, it shall be underlined that even in cryogenic conditions, semiconductors have an intrinsic noise which 


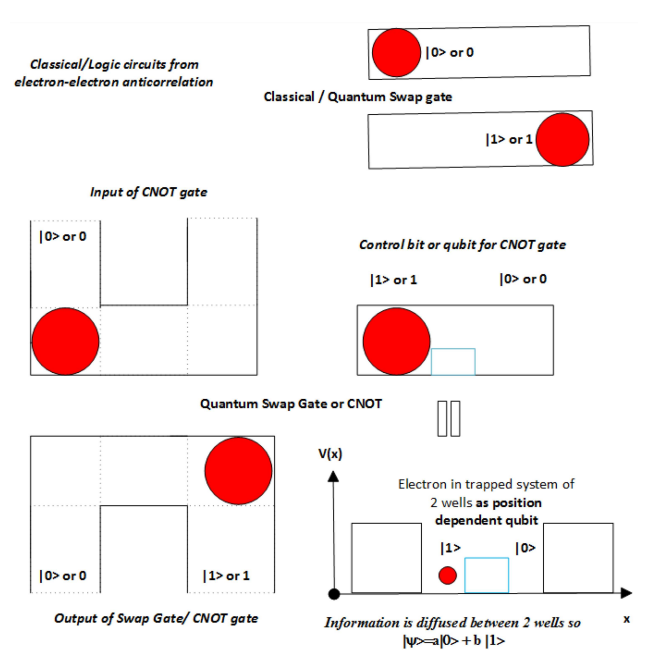

Fig. 1. The Coulomb repulsion principle as the foundation of the electrostatic classical and quantum gates as given by an example of the classical (quantum) inverter (NOT gate) and CNOT gate (controllable NOT gate). The classical gates downsized to nanosize will have features of quantum gates that can be implemented in single-electron devices [4].

is significantly higher than in the case of superconductors. Since IBM Quantum Experience [7, 8] uses low temperature superconductors and a transmon low noise Josephson junction qubit, one can think about the possibility of merging these two quantum computer architectures into one chip. This leads to the necessity of constructing an interface between a Josephson junction and an electrostatic Wannier based qubit. In this consideration, we exclude the role of electron spin that becomes important in $\mathrm{mK}$ regime, while charged based qubits shall be operational at $4 \mathrm{~K}$. The minimum and necessary condition for qubit operation is the partial occupation of two energy levels of quantum dots. This induces oscillations of states occupancies, with the frequency proportional to the energy difference between two energy levels.

The problem of placing electrons in the effective potential is easily solvable by the Schröedinger equation. However, when one considers two or more electrostatically interacting qubits, then integrodifferential equations emerge that are not solvable by analytical methods. Electron movement between two quantum dots is represented by the tightbinding approach which essentially describes energy flow between neighbouring quantum dots and energy localized in each quantum dot. This simplistic approach is quite powerful and will be conducted in the presented paper. It allows to capture the essence of the electrostatic entanglement between coupled position-based qubits. The tightbinding model can be used furthermore in the context of Bogoliubov-de Gennes equations describing the Andreev bound states (ABS) - the essence of the Josephson effect. In fact, the Andreev bound state of a Josephson junction is being modified by the presence of position-based qubit implemented in semiconductor. Such a modification of the Andreev bound state by a single electron in a Wannier semiconductor qubit (position-based qubit) is the subject of this work. It has its scientific, technological and didactic value, while the results might be important for the creation of a hybrid semiconductor-superconducting quantum computer (hybrid semiconductor-superconducting quantum chip) and for quantum information processing in such structures.

\section{Description of position-based qubit in tight-binding model}

In order to refer to the physical situation, we consider a position-based qubit in the tight-binding model [5]. The Hamiltonian is given as

$$
\begin{aligned}
& \hat{H}(t)=\left(\begin{array}{ll}
E_{p 1}(t) & t_{s 12}(t) \\
t_{s 12}^{\dagger}(t) & E_{p 2}(t)
\end{array}\right)_{\left[x=\left(x_{1}, x_{2}\right)\right]}= \\
& \left(E_{1}(t)\left|E_{1}\right\rangle_{t}\left\langle\left. E_{1}\right|_{t}+E_{2}(t) \mid E_{2}\right\rangle\left\langle E_{2}\right|\right)_{\left[E=\left(E_{1}, E_{2}\right)\right]} .
\end{aligned}
$$

and describes the system which consists of two coupled quantum wells as depicted in Fig. 2. In such a situation, we deal with real-valued functions $E_{p 1}(t), \quad E_{p 2}(t)$ and complex-valued functions $t_{s 12}(t)=t_{s}(t)=t_{s r}(t)+\mathrm{i} t_{s i}(t)$, where $t_{s 21}(t)=t_{s 12}^{*}(t)$. It is equivalent to the knowledge of four real valued time-dependent continuous or discontinues functions $E_{p 1}(t), E_{p 2}(t), t_{s r}(t)$ and $t_{s i}(t)$.

The system eigenenergies $E_{1}(t)$ and $E_{2}(t)$, where $E_{2}(t)>E_{1}(t), \Delta E_{p}=E_{p 2}-E_{p 1}$, can be written as

$$
\begin{aligned}
& E_{1}(t)=\frac{E_{p 1}(t)+E_{p 2}(t)-\sqrt{\Delta E_{p}^{2}(t)+4\left|t_{s 12}(t)\right|^{2}}}{2}, \\
& E_{2}(t)=\frac{E_{p 1}(t)+E_{p 2}(t)+\sqrt{\Delta E_{p}^{2}(t)+4\left|t_{s 12}(t)\right|^{2}}}{2}
\end{aligned}
$$

and the corresponding eigenstates $\left|E_{1}(t)\right\rangle$ and $\left|E_{2}(t)\right\rangle$ in the form of

$$
\begin{aligned}
& \left|E_{1}, t\right\rangle=\left[\frac{\Delta E_{p}(t)+\sqrt{\Delta E_{p}^{2}(t)+4\left|t_{s 12}(t)\right|^{2}}}{-\mathrm{i} t_{12}(t)},-1\right]^{T} \\
& \left|E_{2}, t\right\rangle=\left[\frac{-\Delta E_{p}(t)+\sqrt{\Delta E_{p}^{2}(t)+4\left|t_{s 12}(t)\right|^{2}}}{t_{12}^{*}(t)}, 1\right]^{T}
\end{aligned}
$$

The quantum state is a superposition of a state localized at node 1 and 2 and therefore is given as

$$
\begin{gathered}
|\psi\rangle_{[x]}=\alpha(t)|1,0\rangle_{x}+\beta(t)|0,1\rangle_{x}= \\
\alpha(t)\left[\begin{array}{l}
1 \\
0
\end{array}\right]+\beta(t)\left[\begin{array}{l}
0 \\
1
\end{array}\right],
\end{gathered}
$$


where $|\alpha(t)|^{2}\left(|\beta(t)|^{2}\right)$ is the probability of finding a particle at node $1(2)$, respectively. The condition $|\alpha(t)|^{2}+|\beta(t)|^{2}=1$ is fulfilled and obviously $\langle 1,0|| 1,0\rangle_{x}=1=\langle 0,1|| 0,1\rangle_{x}$ and $\langle 1,0|| 0,1\rangle_{x}=$ $0=\langle 0,1|| 1,0\rangle_{x}$. In the Schrödinger formalism, both states $|1,0\rangle_{x}$ and $|0,1\rangle_{x}$ are Wannier functions parametrized by position $x$.

The quantum state of the system evolves according to

$$
\mathrm{i} \hbar \frac{\mathrm{d}}{\mathrm{d} t}|\psi(t)\rangle=\hat{H}(t)|\psi(t)\rangle=E(t)|\psi(t)\rangle .
$$

The general analytic form, being the solution of (5), can be expressed as

$$
\begin{gathered}
|\psi(t)\rangle=\exp \left(\frac{1}{\mathrm{i} \hbar} \int_{t_{0}}^{t} \hat{H}\left(t_{1}\right) \mathrm{d} t_{1}\right)\left|\psi\left(t_{0}\right)\right\rangle= \\
\exp \left(\frac{1}{\mathrm{i} \hbar} \int_{t_{0}}^{t} \hat{H}\left(t_{1}\right) \mathrm{d} t_{1}\right)\left[\begin{array}{c}
\alpha(0) \\
\beta(0)
\end{array}\right]
\end{gathered}
$$

and the density matrix for this state is as follows

$$
\begin{aligned}
& \hat{\rho}(t)=\hat{\rho}^{\dagger}(t)=|\psi(t)\rangle\langle\psi(t)|= \\
& \hat{U}\left(t, t_{0}\right) \hat{\rho}\left(t_{0}\right) \hat{U}\left(t, t_{0}\right)^{-1}= \\
& \exp \left(\frac{1}{\mathrm{i} \hbar} \int_{t_{0}}^{t} \hat{H}\left(t_{1}\right) \mathrm{d} t_{1}\right)\left(\left|\psi\left(t_{0}\right)\right\rangle\left\langle\psi\left(t_{0}\right)\right|\right) \\
& \times \exp \left(-\frac{1}{\mathrm{i} \hbar} \int_{t_{0}}^{t} \hat{H}\left(t_{1}\right) \mathrm{d} t_{1}\right)= \\
& \hat{U}\left(t, t_{0}\right)\left(\begin{array}{cc}
|\alpha(0)|^{2} & \alpha(0) \beta^{*}(0) \\
\beta(0) \alpha(0)^{*} & |\beta(0)|^{2}
\end{array}\right) \hat{U}\left(t, t_{0}\right)^{\dagger} .
\end{aligned}
$$

The initial state is $\left|\psi\left(t_{0}\right)\right\rangle=\left[\alpha^{*}(0), \beta^{*}(0)\right]^{T}$.

\section{Electrostatic interaction of Josephson junction qubit with semiconductor electrostatic qubit}

The state of the Josephson junction is well described by the Bogoliubov-de Gennes (BdGe) equation [9] pointing to the correlation between the electron $(e)$ and holes $(h)$ as

$$
\left(\begin{array}{cc}
H_{0} & \Delta(x) \\
\Delta(x)^{*} & -H_{0}^{\dagger}
\end{array}\right)\left[\begin{array}{l}
u_{n}(x) \\
v_{n}(x)
\end{array}\right]=E_{n}\left[\begin{array}{l}
u_{n}(x) \\
v_{n}(x)
\end{array}\right],
$$

where $H_{0}=-\frac{\hbar^{2}}{2 m} \frac{\mathrm{d}^{2}}{\mathrm{~d} x^{2}}$ is the free electron Hamiltonian with the self-consistency relation

$$
\Delta(x)=\sum_{n}\left(1-2 f\left(E_{n}\right)\right) u_{n}(x) v_{n}(x)^{*} .
$$

Here, $\Delta(x)$ is the superconducting order parameter, $f\left(E_{n}\right)=\left[1+\exp \left(-E_{n} / k_{\mathrm{B}} T\right)\right]^{-1}$ is the Fermi-Dirac distribution function and $u_{n}(x)$ and $v_{n}(x)$ are electron and hole wavefunctions. In the case of a bulk superconductor with a constant superconducting order parameter, we obtain $E_{n}= \pm \sqrt{\left|H_{0}\right|^{2}+|\Delta|^{2}}$. In later considerations,
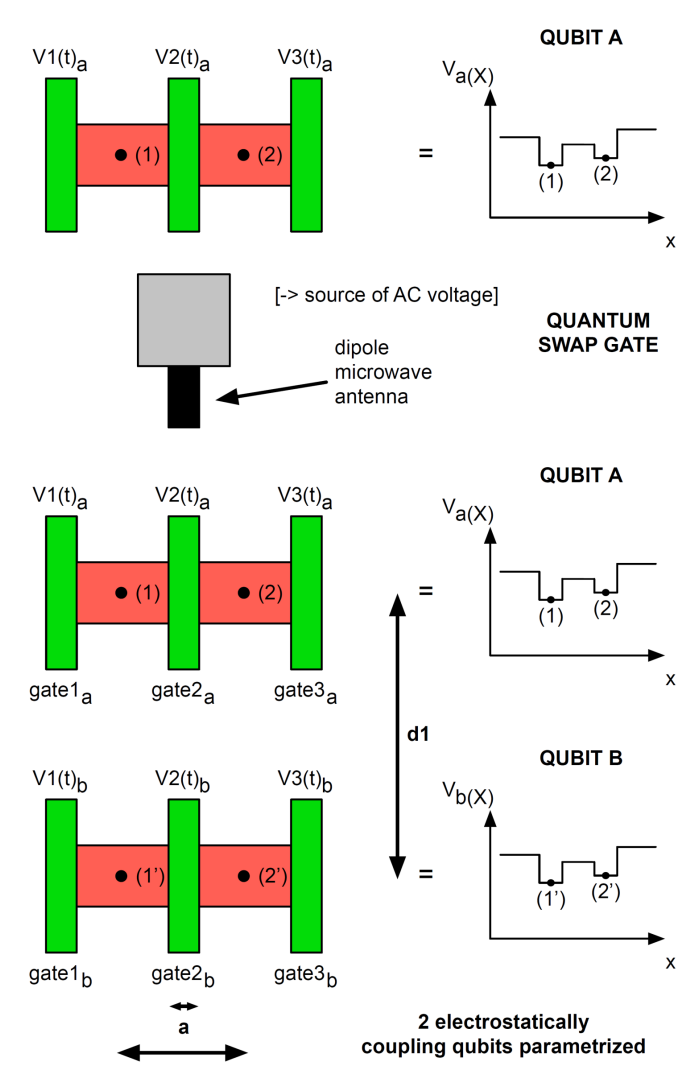

Fig. 2. Electrostatic position-based qubit implemented in CMOS technology [4]. Upper: simplistic representation by a particle localized in two regions of space denoted by nodes (1) and (2). Lower: case of two electrostatically interacting qubits implementing a quantum swap gate. Quantum dynamics are parameterized by the presence of electrons at nodes 1, 2, 1' and 2'.

we will omit the self-consistency relation assuming that the superconducting order parameter is as a step-like function. It should be underlined that the BdGe equation is the mean field equation derived from the BCS theory of superconductivity and naturally it is valid for the case of many particles. A semiconductor single electron line with two nodes can be considered as an electrostatic position-dependent qubit and described with $H_{\text {semi }}=t_{s 12}|1\rangle\left\langle 2\left|+t_{s 21}\right| 2\right\rangle\left\langle 1\left|+E_{p 1}\right| 1\right\rangle\langle 1|+$ $E_{p 2}|2\rangle\langle 2|$.

We refer to the physical situation depicted in Fig. 3. We can express the coupling of two systems assuming four nodes for the electron or hole and two nodes for the electron confined in a semiconductor. Thus, each eigenvector has 16 components $\left(|0\rangle_{e}|1\rangle_{s},|0\rangle_{e}|2\rangle_{s},|1\rangle_{e}|1\rangle_{s}\right.$, $\left.|1\rangle_{e}|2\rangle_{s},|2\rangle_{e}|1\rangle_{s}, \quad|2\rangle_{e}|2\rangle_{s}, \quad|3\rangle_{e}|1\rangle_{s},|2\rangle_{e}|2\rangle_{s}\right)$, $\left(|0\rangle_{h}|1\rangle_{s}, \quad|0\rangle_{h}|2\rangle_{s},|1\rangle_{h}|1\rangle_{s}, \quad|1\rangle_{h}|2\rangle_{s},|2\rangle_{h}|1\rangle_{s}\right.$, $\left.|2\rangle_{h}|2\rangle_{s}, \quad|3\rangle_{h}|1\rangle_{s}, \quad|2\rangle_{h}|2\rangle_{s}\right)$, where $s$ refers to the semiconductor qubit whose quantum state is a superposition of $|1\rangle_{s}$ and $|2\rangle_{s}$. The states $|0\rangle_{e}, \ldots,|3\rangle_{e},|0\rangle_{h}, \ldots,|3\rangle_{h}$ characterize the state of the electron and hole, respectively, in the Andreev bound state of the Josephson junction. It should 


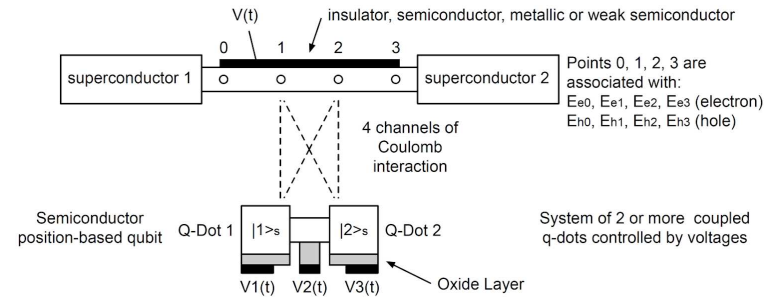

Fig. 3. Superconducting Josephson junction interacting with the semiconductor position-based qubit in the minimalistic tight-binding approach, where the tight-binding BdGe equation describing the Josephson junction is coupled electrostatically to the tight-binding model of semiconductor position-based qubit.

be understood as (i) when the electron moving in the normal (non-superconducting) region between superconductors is reflected as a hole, and (ii) when it comes into the superconducting area and when a hole moving in the normal region is reflected as an electron when it meets a superconductor, etc.
In our case, the quantum state of the system can be written as

$$
\begin{aligned}
& |\psi, t\rangle=\gamma_{1}(t)|0\rangle_{e}|1\rangle_{s}+\gamma_{2}(t)|0\rangle_{e}|2\rangle_{s} \\
& \quad+\gamma_{3}(t)|1\rangle_{e}|1\rangle_{s}+\gamma_{4}(t)|1\rangle_{e}|2\rangle_{s}+\gamma_{5}(t)|2\rangle_{e}|1\rangle_{s} \\
& \quad+\gamma_{6}(t)|2\rangle_{e}|2\rangle_{s}+\gamma_{7}(t)|2\rangle_{e}|1\rangle_{s}+\gamma_{8}(t)|2\rangle_{e}|2\rangle_{s} \\
& \quad+\gamma_{9}(t)|0\rangle_{h}|1\rangle_{s}+\gamma_{10}(t)|0\rangle_{h}|2\rangle_{s}+\gamma_{11}(t)|1\rangle_{h}|1\rangle_{s} \\
& \quad+\gamma_{12}(t)|1\rangle_{h}|2\rangle_{s}+\gamma_{13}(t)|2\rangle_{h}|1\rangle_{s}+\gamma_{14}(t)|2\rangle_{h}|2\rangle_{s} \\
& \quad+\gamma_{15}(t)|2\rangle_{e}|1\rangle_{s}+\gamma_{16}(t)|2\rangle_{h}|2\rangle_{s} .
\end{aligned}
$$

The normalization condition implies $\left|\gamma_{1}(t)\right|^{2}+$ $\left|\gamma_{2}(t)\right|^{2}+\cdots+\left|\gamma_{16}(t)\right|^{2}=1$ for each time $t$. Such a system has 16 eigenenergies. The probability of finding an electron at node 1 under any presence of the electron in a semiconductor qubit at node 1 or 2 is determined by applying projection $\mid\left(\left\langle\left. 1\right|_{e}\left\langle\left. 1\right|_{s}+\left.\left\langle\left. 1\right|_{e}\left\langle\left. 2\right|_{s}\right) \mid \psi, t\right\rangle\right|^{2}\right.\right.\right.$. As a result, we obtain the structures of matrices corresponding to the $H_{0}$ part of the BdGe equation for $A=\{e, h\}$ in the general form

$$
\begin{aligned}
\hat{H}_{0[A]} & =E_{p 2} \hat{I}_{8 \times 8} \\
+ & \left(\begin{array}{cccc}
-\Delta E_{p}+E_{A 0} & t_{s} & t_{A(1,0)} & 0 \\
t_{s}^{*} & E_{A 0} & 0 & t_{A(1,0)} \\
t_{A(1,0)}^{*} & 0 & -\Delta E_{p}+E_{A 1}+\frac{\epsilon_{A} q^{2}}{a} & t_{s} \\
0 & t_{A(1,0)}^{*} & t_{s}^{*} & E_{A 1}+\frac{\epsilon_{A} q^{2}}{b} \\
t_{A(2,0)}^{*} & 0 & t_{A(2,1)}^{*} & 0 \\
0 & t_{A(2,0)}^{*} & 0 & t_{A(2,1)}^{*} \\
t_{A(3,0)}^{*} & 0 & t_{A(3,1)}^{*} & 0 \\
0 & t_{A(3,0)}^{*} & 0 & t_{A(3,1)}^{*}
\end{array}\right.
\end{aligned}
$$$$
\left.\begin{array}{cccc}
t_{A(2,0)} & 0 & t_{A(3,0)} & 0 \\
0 & t_{A(2,0)} & 0 & t_{A(3,0)} \\
t_{A(2,1)} & 0 & t_{A(3,1)} & 0 \\
0 & t_{A(2,1)} & 0 & t_{A(3,1)} \\
-\Delta E_{p}+E_{A 2}+\frac{\epsilon_{A} q^{2}}{b} & t_{s} & t_{A(3,2)} & 0 \\
t_{s}^{*} & E_{A 2}+\frac{\epsilon_{A} q^{2}}{a} & 0 & t_{A(3,2)} \\
t_{A(3,2)}^{*} & 0 & -\Delta E_{p}+E_{3 A} & t_{s} \\
0 & t_{A(3,2)}^{*} & t_{s}^{*} & E_{3 A}
\end{array}\right) .
$$

Parameters $E_{p 1}, E_{p 2}$ (with $\Delta E=E_{p 2}-E_{p 1}$ ) correspond to semiconductor position-based qubits which are coupled with $t_{s}$, while the respective distance between semiconductor qubits and the Josephson junction is $a$ and $b$. Parameter $E_{A k}$ denotes the localized energy of $A=\{$ electron, hole $\}$ at the Josephson junction node $k=0, \ldots, 3$. In turn, $\epsilon_{A}=-1$ if $A=$ hole, or $\epsilon_{A}=1$ if $A=$ electron, what implies $t_{A(k, l)}=-t_{B(k, l)}^{*}$ and $E_{A k}=-E_{B k}$.

Hence, we obtain the form of $\hat{H}_{0[h]}$ which is analogous to the form of $\hat{H}_{(0[e])}$ and, in addition, two other matrices $\hat{\Delta}_{1}, \hat{\Delta}_{2}=\hat{\Delta}_{1}^{\dagger}$, where $\hat{\Delta}_{1}=\operatorname{diag}(\Delta(0), \Delta(0), \Delta(1), \Delta(1), \Delta(2), \Delta(2)$, $\Delta(3), \Delta(3))$. The final structure of the tightbinding Bogoliubov-de Gennes equations including the interaction of the semiconductor qubit with the Josephson junction in the minimalistic way can be described as

$$
\hat{H}_{\mathrm{eff}}=\left(\begin{array}{cc}
\hat{H}_{0[e]} & \hat{\Delta}_{1} \\
\hat{\Delta}_{2} & \hat{H}_{0[h]}
\end{array}\right) .
$$

Similarly as before, we will use the knowledge on quantum state at $t_{0}$. We can evaluate the state by computing its time dependence $\exp \left(\int_{t_{0}}^{t} \frac{1}{\mathrm{i} \hbar} \hat{H}_{\text {ext }}(t) \mathrm{d} t^{\prime}\right)\left|\psi, t_{0}\right\rangle=|\psi, t\rangle$. In fact, it is based on the method already presented in (8). Moreover, we can perform the procedure of heating up or cooling down of the quantum state in the way as it was described before or we can regulate the population of pointed energetic level(s).

In order to work with the most minimalistic tightbinding model of the Josephson junction of Sc-I-Sc (Superconductor-Insulator-Superconductor) we set $\Delta(1)=\Delta(2)=0$ what corresponds to the simplest form of the Andreev bound state in the tunneling Josephson junction. However, in weak-links and in the field-induced Josephson junctions, all diagonal elements are non-zero and $|\Delta|$ has maximum at $\Delta(0)$ and $\Delta(3)$ which can be considered as a superconducting state of bulk superconductors. Quite naturally, the field-induced Josephson junction [9] can have a special profile of dependence 
of superconducting order parameter $\Delta(x)$ on position $x$ with the presence of built-in magnetic fields in the area of junction. It will also have special complex-valued hopping constants for the electron and hole in the area of the superconductor that will incorporate the profile of magnetic field present across the Josephson junction. The specified Hamiltonian describing the electrostatic interface between the superconducting Josephson junction and the semiconductor position-based qubit consists of many parameters. They can be characterized as (i) parameters describing the state of the position-based semiconductor qubit $E_{p 1}, E_{p 2}$, $t_{s}=t_{\mathrm{sr}}+\mathrm{i} t_{i s}$ (four real valued time dependent functions) and (ii) parameters $E_{A k}, \Delta(k), t_{A(k, k \pm 1)}$ (where $A=\{e, h\}$ and $k=0, \ldots, 3$ ) describing the state of the Josephson junction (JJ) as well as (iii) geometrical parameters describing the electrostatic interaction between the semiconductor JJ and the semiconductor qubit by distance $a$ and $b$. It is worth mentioning that electrostatic interaction we take into account occurs only between nodes $1-1 s, 1-2 s, 2-1 s, 2-2 s$, meaning four channels for Coulomb interaction. This assumption greatly simplifies the model so one can find analytical solutions as well. The assumption with four channels of electrostatic interaction is physically justifiable if one assumes that $\Delta(0) \neq 0, \Delta(3) \neq 0$ and $(\Delta(1), \Delta(2)) \rightarrow 0$. Formally, we have omitted the channels of electrostatic interaction as $0-1 s$, $3-1 s, 0-2 s, 3-2 s$. It is commonly known that the superconducting state, especially with strong superconductivity as in the case of a bulk superconductor, is not supporting and shielding itself from the external and internal electrostatic field of certain strength as it naturally protects its ground superconducting macroscopic state.

Having established the mathematical structure describing the electrostatic interaction between the semiconductor position-based qubit and the Josephson junction, we can proceed to analytical and numerical calculations. The first simplification is that $\Delta(1)=\Delta(2)=0$ and $\Delta=\Delta(0)=\Delta(3) \in R$. It means that there is no net electric current flowing via the Josephson junction since the electric current flow imposes the condition of phase difference among superconducting order parameter $\Delta(0)$ and $\Delta(3)$ and in such a case the superconducting order parameter is a complex valued scalar. Also, it implies that there is no magnetic field in our system since magnetic field brings a phase imprint between $\Delta(0)$ and $\Delta(3)$. The second simplification is that $E_{p 1}=E_{p 2}=E_{p}, t_{s} \in R$. The third simplification is that for each node $k=0,1, \ldots 3$ one has $E_{e k}=V=-E_{h k}$ and indicates there is an electron-hole symmetry in the area of ABS, that is in the middle of the Josephson junction. In such a way all hole eigenenergies are corresponding to electron eigenenergies with '-' sign. The last assumption is that the electron or hole hopping in

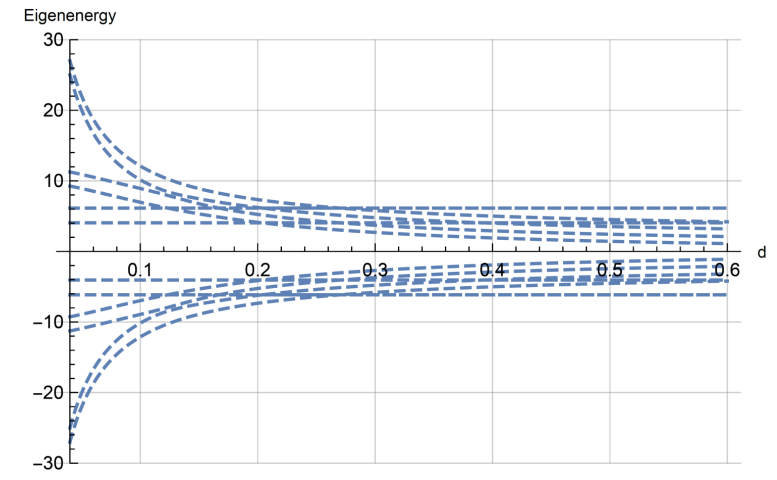

Fig. 4. Eigenenergies of semiconductor qubit coupled to Josephson junction in dependence on distance in tight-binding minimalitic approach.

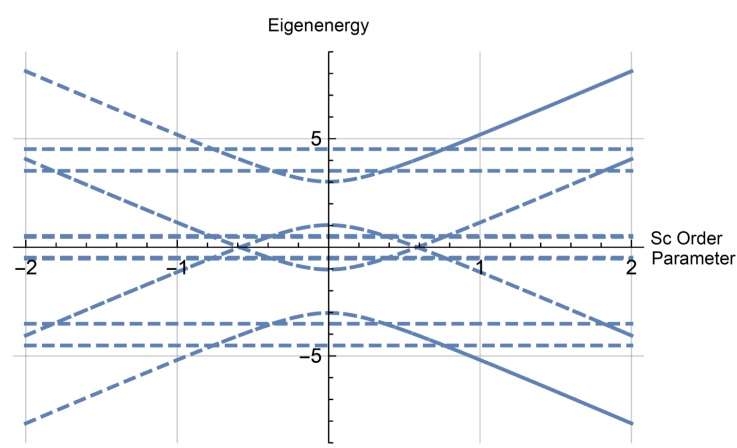

Fig. 5. Eigenenergies of the semiconductor qubit coupled to the Josephson junction in dependence on the superconducting order parameter in a minimalitic approach.

the area of ABS in between the nearest neighbours is such that $t_{e(k, k+1)} \neq 0$ and $t_{h(k, k+1)} \neq 0$ and is 0 otherwise. One can name such a feature of transport in the Josephson junction as diffusive and not ballistic which brings the mathematical simplifications. Establishing these facts we can move into analytical and numerical calculations. Hamiltonian of the physical system has a structure that allows analytic determination of all eigenenergies since the Hamiltonian matrix has many symmetries. In particular, we can obtain the spectrum of eigenenergies in dependence on the distance $d$ as depicted in Fig. 4 and also the spectrum of eigenenergies in dependence on the superconducting order parameter, as seen in Fig. 5.

It is possible to observe the swap of the ground and excited state in the system of the interacting Josephson junction with the semiconductor qubit what implies the existence of the topological phase transition as depicted in Figs. 4-6. Because of that, such a system could be interesting for quantum information processing both in the classical quantum ways and with the use of topological states of matter. Topological states of matter can be controlled by tuning the superconducting order parameter strength with the use of external 


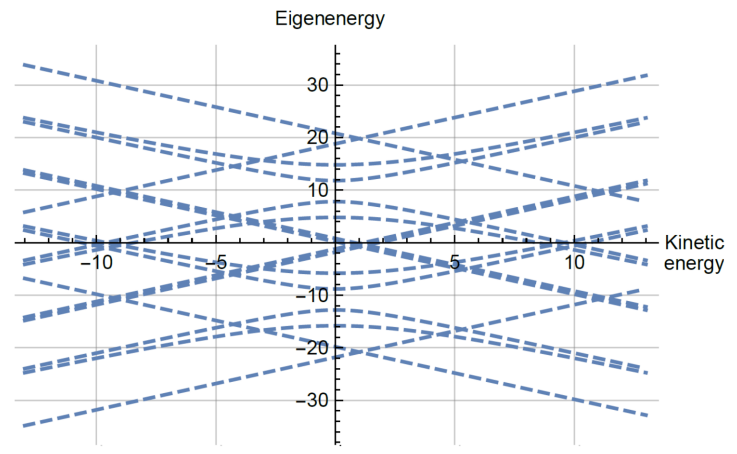

Fig. 6. Different eigenenergies as functions of kinetic energies.

magnetic field or by using an array of semiconductor quantum dots where the distance between a single electron (distributed in a wavepacket) and the Josephson junctions can be changed [10] with the use of electric control acting on the state of a semiconductor qubit.

One of the most interesting features observed in the BdGe tight-binding model is the landscape tuning of eigenenergies by the application of small voltage (below the size $2 e \Delta$ ) to a non-superconducting region of the Josephson junction. In a very real way, the control of this voltage is the control of the chemical potential in the Josephson junction.

In such a case one obtains various features as described in Figs. 6-8. In the described considerations, the spin degree-of-freedom was omitted in the case of the Josephson junction as well as in the case of the semiconductor position-based qubit. However, they could be easily included but it would increase the size of the matrix describing the interaction between the superconductor Josephson junction and the semiconductor electrostatic qubit from $16 \times 16$ to the size $8 \times 4=32$. Thus, one obtains the matrix $32 \times 32$. Adding a strong spinorbit interaction to the Hamiltonian of the Josephson junction under the presence of magnetic field allows to describe the topological Josephson junction. In such a way we can obtain the effective Hamiltonian $32 \times 32$ for the interaction between the semiconductor position-based qubit and the topological Josephson junction in the most minimalistic way.

It should also be underlined that so far we have used the BdGe formalism which is suitable for the mean field theory domain. However, in our case we have considered very special interactions between individual electrons, holes present in the area of the Josephson junction and the specific individual electron present in the area of the semiconductor qubit. The use of the BdGe formalism is therefore the first level of possible approximation and a further more detailed study can be attempted to determine microscopic processes present in the interaction of the Josephson junction with the semiconductor qubit. It is sufficient

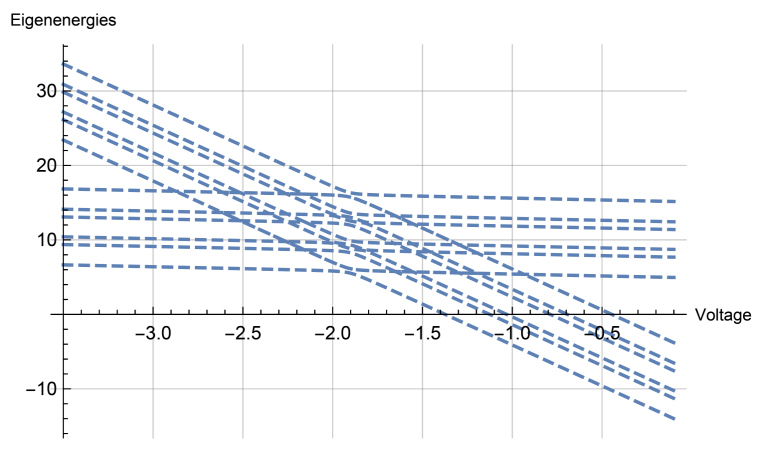

Fig. 7. Tunnning the spectrum of eigenenergies in electrostatic qubit interacting with the Josephson junction while we are changing the chemical potential of the insulator region in the Josephson junction at all nodes $0,1,2$ and 3 at the same time.

to mention that in our case superconductors shall have a relatively small size so we are dealing with a relatively small number of electrons and holes in the non-superconducting area.

More detailed considerations are, however, beyond the scope of this work and require the density functional theory (DFT) methods, etc.

The effective tight-binding Bogoliubov-de Gennes matrix which takes into account the interaction of the semiconductor qubit with the Josephson junction can be at least diagonalized. As a result, two groups of eigenvectors can be distinguished. To show the solutions, the writing convention should be adjusted to make it sufficiently readable. Let us introduce: $\Delta E_{c}=E_{c 2}-E_{c 1}, \Sigma_{t}=t_{j}+t_{s}$, $\delta_{t}=t_{j}-t_{s}$ and with their help we express the energy eigenvalues. They are

$$
\begin{aligned}
& \mathcal{E}_{1}=-\frac{\sqrt{\left(\Delta E_{c}\right)^{2}+\left(2 \delta_{t}\right)^{2}}+E_{c 1}+E_{c 2}}{2}+E_{p}-V, \\
& \mathcal{E}_{2}=\frac{\sqrt{\left(\Delta E_{c}\right)^{2}+\left(2 \delta_{t}\right)^{2}}+E_{c 1}+E_{c 2}}{2}+E_{p}-V \\
& \mathcal{E}_{3}=-\frac{\sqrt{\left(\Delta E_{c}\right)^{2}+\left(2 \Sigma_{t}\right)^{2}}+E_{c 1}+E_{c 2}}{2}+E_{p}-V \\
& \mathcal{E}_{4}=\frac{\sqrt{\left(\Delta E_{c}\right)^{2}+\left(2 \Sigma_{t}\right)^{2}}-\Delta E_{c}}{2}+E_{p}-V \\
& \mathcal{E}_{5}=\frac{-\sqrt{\left(\Delta E_{c}\right)^{2}+\left(2 \delta_{t}\right)^{2}}+E_{c 1}+E_{c 2}}{2}+E_{p}+V, \\
& \mathcal{E}_{6}=\frac{\sqrt{\left(\Delta E_{c}\right)^{2}+\left(2 \delta_{t}\right)^{2}}+E_{c 1}+E_{c 2}}{2}+E_{p}+V, \\
& \mathcal{E}_{7}=\frac{-\sqrt{\left(\Delta E_{c}\right)^{2}+\left(2 \Sigma_{t}\right)^{2}}+E_{c 1}+E_{c 2}}{2}+E_{p}+V, \\
& \mathcal{E}_{8}=\frac{\sqrt{\left(\Delta E_{c}\right)^{2}+\left(2 \Sigma_{t}\right)^{2}}+E_{c 1}+E_{c 2}}{2}+E_{p}+V,
\end{aligned}
$$


In turn, the corresponding eigenvectors are

$$
\begin{aligned}
& |\psi\rangle_{1}= \\
& {\left[\begin{array}{c}
0 \\
0 \\
-1 \\
c_{1,4} \\
c_{1,4} \\
1 \\
0 \\
0 \\
0 \\
0 \\
0 \\
0 \\
0 \\
0 \\
0 \\
0
\end{array}\right]}
\end{aligned}
$$$$
|\psi\rangle_{2}=
$$$$
|\psi\rangle_{3}=
$$$$
|\psi\rangle_{4}=
$$

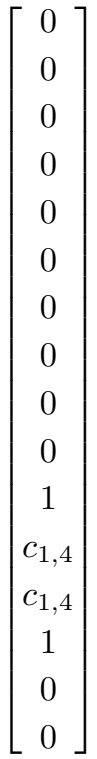

$|\psi\rangle_{4}=$

$\left[\begin{array}{c}0 \\ 0 \\ 0 \\ 0 \\ 0 \\ 0 \\ 0 \\ 0 \\ 0 \\ 0 \\ -1 \\ c_{3,4} \\ -c_{3,4} \\ 1 \\ 0 \\ 0\end{array}\right]$ where

$$
\begin{gathered}
c_{1,4}=c_{2,12}=c_{1,5}=c_{2,13}= \\
-\frac{2 \delta_{t}}{\sqrt{\left(\Delta E_{c}\right)^{2}+\left(2 \delta_{t}\right)^{2}}+\Delta E_{c}}, \\
c_{3,4}=c_{3,5}=c_{4,12}=-c_{4,13}= \\
-\frac{2 \Sigma_{t}}{\sqrt{\left(\Delta E_{c}\right)^{2}+\left(2 \Sigma_{t}\right)^{2}}+\Delta E_{c}},
\end{gathered}
$$

and

$$
|\psi\rangle_{5}
$$$$
|\psi\rangle_{6}=
$$$$
|\psi\rangle_{7}=
$$$$
\left[\begin{array}{c}
0 \\
0 \\
0 \\
0 \\
0 \\
0 \\
0 \\
0 \\
0 \\
0 \\
1 \\
c_{6,4} \\
c_{6,4} \\
1 \\
0 \\
0
\end{array}\right]
$$$$
\left[\begin{array}{c}
0 \\
0 \\
-1 \\
c_{6,4} \\
-c_{6,4} \\
1 \\
0 \\
0 \\
0 \\
0 \\
0 \\
0 \\
0 \\
0 \\
0 \\
0
\end{array}\right]
$$

where

$$
\begin{gathered}
c_{6,4}=-c_{6,5}=c_{5,12}=c_{5,13}= \\
\frac{2 \delta_{t}}{\sqrt{\left(\Delta E_{c}\right)^{2}+\left(2 \delta_{t}\right)^{2}}-\Delta E_{c}}, \\
c_{8,4}=c_{8,5}=c_{7,12}=-c_{7,13}= \\
\frac{2 \Sigma_{t}}{\sqrt{\left(\Delta E_{c}\right)^{2}+\left(2 \Sigma_{t}\right)^{2}}-\Delta E_{c}} .
\end{gathered}
$$

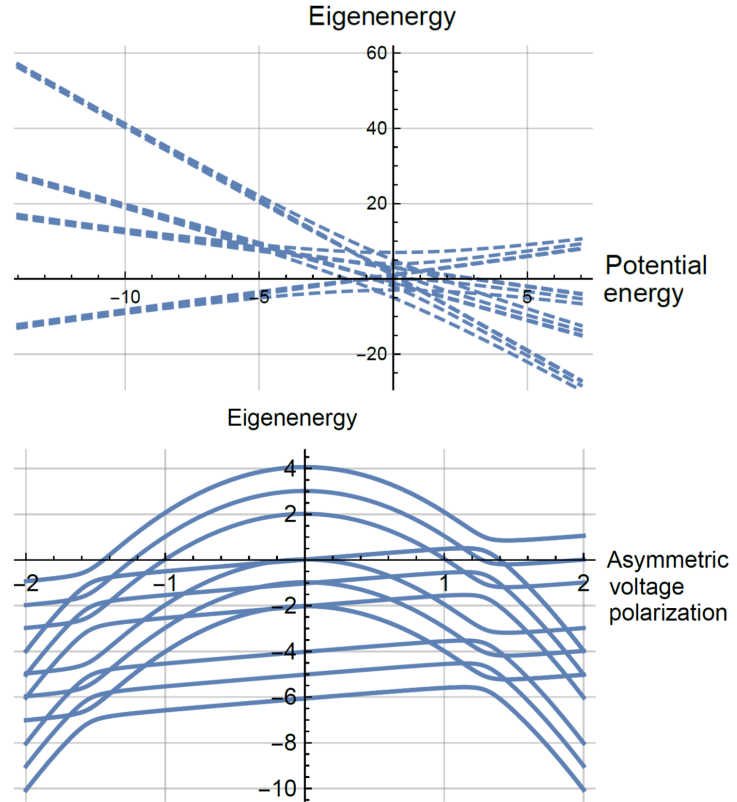

Fig. 8. Different eigenenergies as functions of tuning voltages.

The coefficients $c_{\mathrm{r}, \mathrm{p}}$ should be read as the r-th eigenvector with $\mathrm{p}-$ position in the $\mathrm{r}$ eigenvector.

We recognize that states $\left|\psi_{1}\right\rangle, \ldots,\left|\psi_{8}\right\rangle$ are entangled due to non-zero Coulomb interaction between the semiconductor position-based qubit and the Josephson junction superconducting qubit. However, other states are not entangled and are given as a tensor product of two non-interacting quantum systems (which is equivalent to the semiconductor qubit and superconducting qubit at sufficiently high distances) and which is also reflected in the lack of dependence of eigenenergies on the Coulomb energy. A quick evaluation of energies involved in the BdGe tight-binding model is made in Table I. For the sake of comparison, the length of the Josephson junction was assumed to be $100 \mathrm{~nm}$ (smaller than the superconducting coherence length for most low temperature BCS superconductors) and the size of the position-based qubit was assumed to be $100 \mathrm{~nm}$ too (the most recent technologies allow for the reduction of this size to $3 \mathrm{~nm}$ ).

From the brief analysis conducted in Table I it can be concluded that it is desirable to use long Josephson junctions that are in close proximity to a semiconductor qubit to ensure that the energy of kinetic excitations can be as small as possible and hence the Coulomb interaction will become a stronger tuning factor. Using strong superconductors would compensate the electrostatic qubitqubit interaction and thus it is not desirable and, consequently, one shall stick to the BCS superconductors. Operational temperature shall be kept in the $\mathrm{mK}$ regime.

We continue the analysis of energy eigenstates of the semiconductor qubit-Josephson junction 
Scaling of tight-binding model parameters with geometry of interface between semiconductor quantum dot qubit and Josephson junction qubit in symmetric case.

\begin{tabular}{c|c|c}
\hline \hline $\begin{array}{c}\text { BdGe } \\
\text { parameter }\end{array}$ & \multicolumn{1}{|c}{ Mathematical formula } & \multicolumn{1}{c}{$\begin{array}{c}\text { Physical value } \\
(\Delta x=d=100 \mathrm{~nm}, n=m=1)\end{array}$} \\
\hline$t_{j}$ & $\frac{\hbar^{2}(2 n+1)}{2 m_{e}, \mathrm{sc}}\left(\frac{2 \pi}{d_{\mathrm{sem}-\mathrm{qbit}}}\right)^{2}=\frac{\hbar^{2}(2 n+1)}{2 m_{e}, \mathrm{sc}}\left(\frac{2 \pi}{\Delta x}\right)^{2}$ & $3 \times 5.938 \mathrm{meV}=17.814 \mathrm{meV}$ \\
$t_{s}$ & $\frac{\hbar^{2}(2 k+1)}{2 m_{e, \mathrm{semi}}}\left(\frac{2 \pi}{d_{\mathrm{JJ}}}\right)^{2}=\frac{\hbar^{2}(2 k+1)}{2 m_{e, \mathrm{semi}}}\left(\frac{2 \pi}{\Delta x}\right)^{2}$ & $3 \times 5.938 \mathrm{meV}=17.814 \mathrm{meV}$ \\
$E_{c 1}$ & $\frac{q^{2}}{a}=\frac{1}{4 \pi \epsilon_{0}} \frac{q^{2}}{d}=\frac{e^{2}}{4 \pi \epsilon_{0} \Delta x}$ & $0.145 \mathrm{meV}$ \\
$E_{c 2}$ & $\frac{q^{2}}{b}=\frac{1}{4 \pi \epsilon_{0}} \frac{q^{2}}{\sqrt{d^{2}+(2 \Delta x)^{2}}}=\frac{e^{2}}{4 \sqrt{5} \pi \epsilon_{0} \Delta x}$ & $0.0659 \mathrm{meV}$
\end{tabular}

system. The remaining states form a group of nonentangled eigenstates $\left|\psi_{9}\right\rangle, \ldots,\left|\psi_{16}\right\rangle$. To present them explicitly we use the following expression: $\xi_{ \pm}=\left[V \pm \sqrt{\left(\Delta^{2}+V^{2}\right)}\right] / \Delta$. The eigenstates are thus

$$
\begin{aligned}
& |\psi\rangle_{9}= \\
& |\psi\rangle_{10}= \\
& |\psi\rangle_{11}=\quad|\psi\rangle_{12}= \\
& {[} \\
& \begin{array}{l}
0 \\
0 \\
0
\end{array} \\
& 0 \\
& \begin{array}{l}
0 \\
0
\end{array} \\
& 0 \\
& -\mathrm{e}^{\mathrm{i} \phi_{2}} \xi_{-} \\
& \mathrm{e}^{\mathrm{i} \phi_{2} \xi_{-}} \\
& 0 \\
& 0 \\
& 0 \\
& 0 \\
& 0 \\
& 0 \\
& \text { - } \\
& -1 \\
& {\left[\begin{array}{c}
-\mathrm{e}^{\mathrm{i} \phi_{1}} \xi_{-} \\
\mathrm{e}^{\mathrm{i} \phi_{1}} \xi_{-} \\
0 \\
0 \\
0 \\
0 \\
0 \\
0 \\
-1 \\
1 \\
0 \\
0 \\
0 \\
0 \\
0 \\
0
\end{array}\right]} \\
& {\left[\begin{array}{c}
0 \\
0 \\
0 \\
0 \\
0 \\
0 \\
\mathrm{e}^{\mathrm{i} \phi_{2}} \xi_{-} \\
\mathrm{e}^{\mathrm{i} \phi_{2}} \xi_{-} \\
0 \\
0 \\
0 \\
0 \\
0 \\
0 \\
1 \\
1
\end{array}\right]} \\
& {\left[\mathrm{e}^{\mathrm{i} \phi_{1}} \xi_{-}\right]} \\
& \mathrm{e}^{\mathrm{i} \phi_{1}} \xi_{-} \\
& 0 \\
& 0 \\
& 0 \\
& 0 \\
& 0 \\
& 0 \\
& 1 \\
& 1 \\
& 0 \\
& 0 \\
& 0 \\
& 0 \\
& \begin{array}{l}
0 \\
0
\end{array} \\
& |\psi\rangle_{13}= \\
& |\psi\rangle_{14}= \\
& |\psi\rangle_{15}= \\
& |\psi\rangle_{16}= \\
& {[} \\
& 0 \\
& \begin{array}{l}
0 \\
0
\end{array} \\
& -\mathrm{e}^{\mathrm{i} \phi_{2}} \xi_{+} \\
& \mathrm{e}^{\mathrm{i} \phi_{2} \xi_{+}} \\
& 0 \\
& {\left[-\mathrm{e}^{\mathrm{i} \phi_{1}} \xi_{+}\right]} \\
& {\left[\begin{array}{l}
0 \\
0
\end{array}\right.} \\
& 0 \\
& 0 \\
& 0 \\
& 0 \\
& 0 \\
& 0 \\
& 0 \\
& -1 \\
& 0 \\
& 0 \\
& 0 \\
& 0 \\
& 0 \\
& -1 \\
& 1 \\
& 1 \\
& {\left[\begin{array}{c}
0 \\
0 \\
0 \\
0 \\
0 \\
\mathrm{e}^{\mathrm{i} \phi_{2} \xi_{+}} \\
\mathrm{e}^{\mathrm{i} \phi_{2}} \xi_{+} \\
0 \\
0 \\
0 \\
0 \\
0 \\
0 \\
1 \\
1
\end{array}\right]} \\
& {\left[\begin{array}{l}
\mathrm{e}^{\mathrm{i} \phi_{1}} \xi_{+} \\
\mathrm{e}^{\mathrm{i} \phi_{1}} \xi_{+}
\end{array}\right]} \\
& 0 \\
& 0 \\
& 0 \\
& 0 \\
& 0 \\
& 0 \\
& 0 \\
& 1 \\
& 1 \\
& 0 \\
& 0 \\
& 0 \\
& 0 \\
& 0 \\
& 0
\end{aligned}
$$

Of course, some eigenvalues are degenerate:

$$
\begin{aligned}
& \mathcal{E}_{9}=\mathcal{E}_{10}=-\sqrt{\Delta^{2}+V^{2}}+E_{p}-t_{s}, \\
& \mathcal{E}_{11}=\mathcal{E}_{12}=-\sqrt{\Delta^{2}+V^{2}}+E_{p}+t_{s}, \\
& \mathcal{E}_{13}=\mathcal{E}_{14}=\sqrt{\Delta^{2}+V^{2}}+E_{p}-t_{s} \\
& \mathcal{E}_{15}=\mathcal{E}_{16}=\sqrt{\Delta^{2}+V^{2}}+E_{p}+t_{s}
\end{aligned}
$$

Conducted considerations can be extended further with the introduction of a noise model for Wannier semiconductor qubits as presented in [11].

\section{Conclusions}

This work describes the elementary but still meaningful model of electrostatic interface between the electrostatic position-based qubit implemented in coupled semiconductor quantum dots (as present in CMOS technology) coupled to the Josephson junction qubit. The emergence of the electrostatic entanglement was shown which is the example of an interface between a superconducting quantum computer and a semiconductor quantum computer.

The obtained results are meaningful for the development of single-electron electrostatic quantum neural networks, quantum gates, such as CNOT, SWAP, Toffoli and Fredkin gates as well as any other types of quantum gates with $N$ inputs and $M$ outputs. Single-electron semiconductor devices can be attractive from the point of view of power consumption and they can approach similar performance as Rapid Single Quantum Flux superconducting circuits [4] having much smaller dimensions than the superconducting circuits. In the conducted computations, the spin degree-of-freedom was neglected. However, it can be added in a straightforward way doubling the size of a Hilbert space. The obtained results allow us to obtain the entanglement of qubit A (for example) using biparticle Von Neumann entropy $S(t)_{\text {A }}$ of qubit A in two electrostatically interacting qubits with time as given by

$$
S(t)=-\operatorname{Tr}\left[\hat{\rho}_{A}(t)\left(\log \left(\hat{\rho}_{\mathrm{A}}(t)\right)\right)\right],
$$

where $\operatorname{Tr}[]$ is the matrix trace operator and $\hat{\rho}_{\mathrm{A}}$ is the reduced density matrix of A qubit after the presence of B qubit was traced out. The obtained results can be mapped to the Schrödinger formalism [12] in order to obtain higher accuracy and 
resolution in the description of quantum state dynamics. One can use the obtained results in the determination of quantum transport in the single electron devices or arbitrary topology which can be helpful in optimization of device functionality and controlling sequences shaping the electron confinement potential. Topological phase transitions, as described by [13-15], are expected to take place in arrays of coupled electrostatic qubits due to the similarity of tight-binding applied in the semiconductor coupled quantum well model to the Josephson model in Cooper pair box superconducting qubits. All results are straightforward enough to be generalized for electrons and holes confined in a net of coupled quantum dots (which changes only the sign of electrostatic energy so $q^{2} \rightarrow-q^{2}$ ) under the assumption that recombination processes do not occur. What is more, the interaction between the electrostatic position-based qubit and the Josephson junction was formulated and solved in the tightbinding model. In quite a straightforward way, one obtains the electrostatically coupled networks of graphs interacting with the single Josephson junction in an analytical way. It shall have its importance in the development of the interface between a semiconductor CMOS quantum computer and an already developed superconducting computer.

\section{Acknowledgments}

This work was supported by Science Foundation Ireland under Grant 14/RP/I2921. We would like to thank Erik Staszewski (erik.staszewski@ucd.ie) for his assistance in graphical design of figures. Some of the considerations are described on the Quantum Hardware Systems YouTube channel [16]. The first idea of constructing a semiconductor qubit-superconducting qubit interface was proposed by the first author of this paper and also expressed in [4]. Preliminary work on the electrostatic entanglement was presented in $[5,10,17]$.

\section{References}

[1] K.K. Likharev, Proc. IEEE 87, 606 (1999).

[2] D. Leipold, "Controlled Rabi Oscillations as Foundation for Entangled Quantum Aperture Logic", Seminar at UC Berkley Quantum Labs, 25th July 2018.
[3] P. Giounanlis, E. Blokhina, K. Pomorski, D.R. Leipold, R.B. Staszewski, IEEE Access 7, 49262 (2019).

[4] K. Pomorski, P. Giounanlis, E. Blokhina, D. Leipold, P. Peczkowski, R.B. Staszewski, in: Superconductivity and Particle Accelerators 2018, Vol. 11054, Eds. D. Bocian, R.S. Romaniuk, SPIE, 2019.

[5] K. Pomorski, P. Giounanlis, E. Blokhina, D. Leipold, R.B. Staszewski, Semicond. Sci. Technol. 34125015 (2019).

[6] I. Bashir, M. Asker, C. Cetintepe et al., in: ESSCIRC 2019 - IEEE 45th European Solid State Circuits Conference (ESSCIRC), Cracow, 2019, p. 125.

[7] IBM Quantum Experience.

[8] Qiskit — Open-source framework.

[9] K. Pomorski, P. Prokopow, Phys. Status Solidi B 249, 1805 (2012).

[10] K. Pomorski, R.B. Staszewski, arXiv:1907.03180v3 (2019).

[11] K. Pomorski, in: Proc. Future Technologies Conf. (FTC) 2020, Vol. 2, Springer, Cham (Switzerland), p. 31.

[12] H.Q. Xu, Phys. Rev. B 66, 165305 (2002).

[13] M.S. Choi, J. Yi, M.Y. Choi, J. Choi, S.I. Lee, Phys. Rev. B 57, R716 (1998).

[14] S. Sachdev, Quantum Phase Transitions, Cambridge Univ. Press, 2011.

[15] D. Maile, S. Andergassen, W. Belzig, Phys. Rev. B 97, 155427 (2018).

[16] K. Pomorski, "Semiconductor Qubits and Hybrid Semiconductor-Superconductor Quantum Computer (Part 1)" at YouTube channel: Quantum Hardware Systems, 2020.

[17] B. Szafran, Phys. Rev. B 101, 075306 (2020). 